\title{
Convolution by means of bilinear maps
}

\author{
Oscar Blasco
}

\begin{abstract}
Given three complex Banach spaces $X, Y, Z$ and $u: X \times Y \rightarrow Z$ a bounded bilinear map. For $f(z)=\sum_{n=0}^{m} x_{n} z^{n}$ where $x_{n} \in X$ and $g(z)=$ $\sum_{n=0}^{k} y_{n} z^{n}$ where $y_{n} \in X$, we define the $u$-convolution of $f$ an $g$ as the polynomial given by $f *_{u} g(z)=\sum_{n=0}^{\min \{m, k\}} u\left(x_{n}, y_{n}\right) z^{n}$.

It is shown that whenever $X$ and $Y$ veryfies the vector-valued analogue of certain inequalities due to Littlewood and Paley for Hardy spaces we have that if $1 \leq p_{1} \leq 2,1 \leq p_{2} \leq \infty$ such that $\frac{1}{p_{1}}+\frac{1}{p_{2}} \geq 1$ and $1 \leq p, q \leq \infty$ are such that $\frac{1}{p}=\frac{1}{p_{1}}+\frac{1}{p_{2}}-1$ and $\frac{1}{q}=\frac{1}{2}+\frac{1}{\max \left\{p_{2}, 2\right\}}$ then there exists a
\end{abstract} constant $C>0$ such that

$$
\left(\int_{0}^{1}(1-r)^{q-1}\left(\int_{-\pi}^{\pi}\left\|(f * u g)^{\prime}\left(r e^{i t}\right)\right\|^{p} \frac{d t}{2 \pi}\right)^{\frac{q}{p}} d r\right)^{\frac{1}{q}} \leq C\|u\|\|\mid f\|_{p_{1}}\|g\|_{p_{2}}
$$

for any $f(z)=\sum_{n=0}^{N} x_{n} z^{n} \in \mathcal{P}(X)$ and $g(z)=\sum_{n=0}^{M} y_{n} z^{n} \in \mathcal{P}(Y)$.

Several applications of this result are obtained.

\section{Introduction.}

In this paper we are going to consider a very general notion of convolution that makes sense for vector valued functions and which extends several situations existing in the literature. Our objetive will be to get some improvements of the well-known Young's theorem on convolutions in the setting of analytic functions.

Let us first use the notation of $L^{p}(\mathbb{T}, X)$ for the $p$-integrable Bochner functions and recall that the notion of convolution has been considered in different context.

Given $f, g \in L^{1}(\mathbb{T})$ the convolution is defined as

$$
f * g(t)=\int_{-\pi}^{\pi} f\left(e^{i s}\right) \cdot g\left(e^{i(t-s)}\right) \frac{d s}{2 \pi} \in L^{1}(\mathbb{T}) .
$$

Same notion makes sense for $f, g \in L^{1}(\mathbb{T}, A)$ where $A$ is a Banach algebra and the product is understood in the sense of the algebra, and then $f * g \in L^{1}(\mathbb{T}, A)$.

1991 Mathematics Subject Classification. Primary 46E40, Secondary 42A85.

Key words and phrases. Vector valued Hardy spaces, Geometry of Banach spaces.

Partially supported by a grant from D.G.I.C.Y.T. PB95-0291 
Now for a general Banach space $X$ we still can consider $f \in L^{1}(\mathbb{T})$ and $g \in$ $L^{1}(\mathbb{T}, X)$ and define

$$
f * g(t)=\int_{-\pi}^{\pi} f\left(e^{i s}\right) \cdot g\left(e^{i(t-s)}\right) \frac{d s}{2 \pi} \in L^{1}(\mathbb{T}, X) .
$$

Even if $f \in L^{1}(\mathbb{T}, X)$ and $g \in L^{1}\left(\mathbb{T}, X^{*}\right)$ and define

$$
f * g(t)=\int_{-\pi}^{\pi}<f\left(e^{i s}\right), g\left(e^{i(t-s)}\right)>\frac{d s}{2 \pi} \in L^{1}(\mathbb{T}),
$$

where $<x, x^{*}>$ stands for the dualilty pairing.

A further step consists of taking $X, Y$ Banach spaces and denote by $L(X, Y)$ the space of linear continuous operators. Now given $f \in L^{1}(\mathbb{T}, L(X, Y))$ and $g \in$ $L^{1}(\mathbb{T}, X)$ and define

$$
f * g(t)=\int_{-\pi}^{\pi} f\left(e^{i s}\right)\left(g\left(e^{i(t-s)}\right) \frac{d s}{2 \pi} \in L^{1}(\mathbb{T}, Y) .\right.
$$

All of them are very particular instances of the following general principle:

Given $X, Y, Z$ Banach spaces and a bounded bilinear map $u: X \times Y \rightarrow Z$ and given $f \in L^{1}(\mathbb{T}, X)$ and $g \in L^{1}(\mathbb{T}, Y)$ and define

$$
f *_{u} g(t)=\int_{-\pi}^{\pi} u\left(f\left(e^{i s}\right), g\left(e^{i(t-s)}\right) \frac{d s}{2 \pi} \in L^{1}(\mathbb{T}, Z) .\right.
$$

For the use of this notion for analytic functions the reader is referred to [AB2] and to $[\mathbf{A}]$ in the setting of vector-valued distributions. Here we concentrate ourselves in our applications on the following bilinear maps:

(1) Given any measure $\mu$ and $1 \leq p_{1}, p_{2} \leq \infty$ such that $\frac{1}{p_{1}}+\frac{1}{p_{2}}=\frac{1}{p}$. Hölder's inequality gives that $u: L^{p_{1}}(\mu) \times L^{p_{2}}(\mu) \rightarrow L^{p}(\mu)$ given by $u(f, g)=f . g$ is a bounded bilinear map.

(2) Given $1 \leq p_{1}, p_{2} \leq \infty$ such that $\frac{1}{p_{1}}+\frac{1}{p_{2}} \geq 1$ and $\frac{1}{p}=\frac{1}{p_{1}}+\frac{1}{p_{2}}-1$. Young's theorem gives that $u: L^{p_{1}}\left(\mathbb{R}^{n}\right) \times L^{p_{2}}\left(\mathbb{R}^{n}\right) \rightarrow L^{p}\left(\mathbb{R}^{n}\right)$ given by $u(f, g)=f * g$ is a bounded bilinear map.

(3) Given three Banach spaces $X, Y, Z$, then $u: L(X, Y) \times L(Y, Z) \rightarrow L(X, Z)$ given by the composition $u(T, S)=S T$ defines a bounded bilinear map.

(4) Given two Banach spaces $X, Y$, and denoting $X \hat{\otimes} Y$ the projective tensor product, then the natural embedding $u: X \times Y \rightarrow X \hat{\otimes} Y$ given by $u(x, y)=x \otimes y$ defines a bounded bilinear map.

Note that all the examples presented for motivation run into this particular cases.

In this paper we shall consider this general convolution in the setting of vectorvalued analytic functions. For this situation the definition is as follows (see [AB2]):

Given three complex Banach spaces $X, Y, Z$ and $u: X \times Y \rightarrow Z$ a bounded bilinear map. For $f(z)=\sum_{n=0}^{m} x_{n} z^{n}$ where $x_{n} \in X$ and $g(z)=\sum_{n=0}^{k} y_{n} z^{n}$ where $y_{n} \in X$, we define the $u$-convolution of $f$ an $g$ as the polynomial given by

$$
f *_{u} g(z)=\sum_{n=0}^{\min \{m, k\}} u\left(x_{n}, y_{n}\right) z^{n} .
$$

Throughout the paper we denote by $\mathcal{P}(X)$ and $\mathcal{H}(X)$ the set of polynomials and holomorphic functions from the unit disc $\mathbb{D}$ into a complex Banach space $X$ 
respectively. As usual, we write $M_{p}(f, r)=\left(\frac{1}{2 \pi} \int_{-\pi}^{\pi}\left\|f\left(r e^{i t}\right)\right\|^{p} d t\right)^{\frac{1}{p}}$, and $H^{p}(X)$ stands for the Hardy space of $X$-valued functions, understood as the subspace of $L^{p}(\mathbb{T}, X)$ of those functions $f$ with $\hat{f}(n)=0$ for $n<0$, or in other words the closure of polynomials under the norm given by $\sup _{0<r<1} M_{p}(f, r)$.

The ideas appearing in this paper were first introduced in the paper $[\mathbf{A B 2}]$ and were motivated by the previous papers $[\mathbf{B 1}, \mathbf{A B 1}$.

It is clear that if $1 \leq p_{1}, p_{2} \leq \infty$ such that $\frac{1}{p_{1}}+\frac{1}{p_{2}} \geq 1$ and $\frac{1}{p}=\frac{1}{p_{1}}+\frac{1}{p_{2}}-1$ and $u: X \times Y \rightarrow Z$ is a bounded bilinear map then given $f \in L^{p_{1}}(\mathbb{T}, X)$ and $g \in L^{p_{2}}(\mathbb{T}, Y)$ then $f *_{u} g \in L^{p}(\mathbb{T}, Z)$. Moreover

$$
\left\|f *_{u} g\right\|_{p} \leq\|f\|_{p_{1}}\|g\|_{p_{2}} .
$$

Our aim is to show that in the setting of vector-valued Hardy spaces this result can be improved. We shall show that under certain assumptions on the Banach spaces $X$ and $Y$, if we start with functions $f \in H^{p_{1}}(\mathbb{T}, X)$ and $g \in H^{p_{2}}(\mathbb{T}, Y)$ then $f *_{u} g$ belongs not only to $H^{p}(\mathbb{T}, Z)$ but even to certain smaller space.

To understand the correct setting for the improvement of Young's result we are looking for, let us recall the following two important inequalities in the theory of Hardy spaces.

It was shown by Littlewood and Paley (see $[\mathbf{L P}]$ ) that for $2 \leq p<\infty$ there exists a constant $C>0$ such that for all $f \in H^{p}$,

$$
\left(\int_{0}^{1}(1-r)^{p-1} M_{p}^{p}\left(f^{\prime}, r\right) d r\right)^{\frac{1}{p}} \leq C\|f\|_{p} .
$$

It was shown by Hardy and Littlewood (see [HL3]) that for $1 \leq p \leq 2$ there exists a constant $C>0$ such that for all $f \in H^{p}$,

$$
\left(\int_{0}^{1}(1-r) M_{p}^{2}\left(f^{\prime}, r\right) d r\right)^{\frac{1}{2}} \leq C\|f\|_{p} .
$$

These two result lead, among other things, to the consideration of the following spaces: For $1 \leq p, q \leq \infty$ and for $0<\alpha$ we shall denote by $H^{p, q, \alpha}(X)$ the space given by those functions in $\mathcal{H}(X)$ such that

$$
\int_{0}^{1}(1-r)^{\alpha q-1} M_{p}^{q}(f, r) d r<\infty,
$$

with the obvious modification for the case $q=\infty$ (see Section 1$)$.

These spaces were considered first (in the scalar valued case) by Hardy-Littlewood and Flett (see $[$ HL1, HL2, F1, F2]) and then for many other authors. With this notation the previous results say that

$$
f \in H^{p} \Longrightarrow f^{\prime} \in H^{p, \max \{p, 2\}, 1} .
$$

We shall need to deal with Banach spaces $X$ (see Section 1), shortly written $X \in(H)_{p}$ where $(0.3)$ holds for functions in $H^{p}(X)$.

Our main result is now as follows:

Main Theorem. Let $1 \leq p_{1} \leq 2,1 \leq p_{2} \leq \infty$ such that $\frac{1}{p_{1}}+\frac{1}{p_{2}} \geq 1$. Let $u: X \times Y \rightarrow Z$ be a bounded bilinear map where $X, Y, Z$ are complex Banach spaces. Let $1 \leq p, q \leq \infty$ are such that $\frac{1}{p}=\frac{1}{p_{1}}+\frac{1}{p_{2}}-1$ and $\frac{1}{q}=\frac{1}{2}+\frac{1}{\max \left\{p_{2}, 2\right\}}$. 
Assume $X \in(H)_{p_{1}}$ and $Y \in(H)_{p_{2}}$. Then if $f \in H^{p_{1}}(X), g \in H^{p_{2}}(Y)$ then $\left(f *_{u} g\right)^{\prime} \in H^{p, q, 1}(Z)$.

In other words, there exists a constant $C>0$ such that

$$
\left\|\left(f *_{u} g\right)^{\prime}\right\|_{p, q, 1} \leq C\|u\|\|f\|_{p_{1}}\|g\|_{p_{2}}
$$

for any $f(z)=\sum_{n=0}^{N} x_{n} z^{n} \in \mathcal{P}(X)$ and $g(z)=\sum_{n=0}^{M} y_{n} z^{n} \in \mathcal{P}(Y)$.

The paper is divided into three sections. In section 1 we introduce the convolution, the property $(H)_{p}$ corresponding to the vector-valued formulation of $(0.3)$ and give some general properties of the spaces $H^{p, q, \alpha}(X)$. In the second section we prove the main theorem and present some corollaries of it. Finally Sections 3,4 and 5 are devoted to give some applications for different bilinear maps. The reader is also referred to $[\mathbf{B 1}, \mathbf{A B 1}, \mathbf{A B 2}]$ for results of similar nature and other applications.

As usual, throughout the paper, the constant $C$ may vary from line to line.

\section{Preliminaries.}

Definition 1.1. (see [AB2]) Let $u: X \times Y \rightarrow Z$ be a bounded bilinear map. Let $f \in \mathcal{H}(X)$ and $g \in \mathcal{H}(Y)$ given by $f(z)=\sum_{n=0}^{\infty} x_{n} z^{n}$ and $g(z)=\sum_{n=0}^{\infty} y_{n} z^{n}$. We define the u-convolution of $f$ an $g$ as the function in $\mathcal{H}(Z)$ given by

$$
f *_{u} g(z)=\sum_{n=0}^{\infty} u\left(x_{n}, y_{n}\right) z^{n}
$$

Definition 1.2. (see $[\mathbf{A B 2}])$ Let $1 \leq p<\infty$. A complex Banach space $X$ is said to have property $(H)_{p}$, to be denoted $X \in(H)_{p}$, if there exists a constant $C>0$ such that

$$
\left(\int_{0}^{1}(1-r)^{\max \{2, p\}-1} M_{p}^{\max \{2, p\}}\left(f^{\prime}, r\right) d r\right)^{\frac{1}{\max \{2, p\}}} \leq C\|f\|_{p}
$$

for any polynomial $f \in \mathcal{P}(X)$.

REMARK 1.1. The property $(H)_{1}$ was already defined and studied in [B1], denoted there by $(H L)$ and then again in [AB2].

REMARK 1.2. The property $(H)_{\infty}$ would mean

$$
M_{\infty}\left(f^{\prime}, r\right) \leq C \frac{M_{\infty}(f, r)}{1-r}
$$

which holds true for any Banach space.

Definition 1.3. Let $1 \leq p \leq \infty, 1 \leq q<\infty$ and $0<\alpha$. We shall denote by $H^{p, q, \alpha}(X)$ the space of functions $f \in \mathcal{H}(X)$ such that

$$
(1-r)^{\alpha} M_{p}(f, r) \in L^{q}\left(\frac{d r}{1-r}\right),
$$

and set $\|f\|_{p, q, \alpha}=\left(\int_{0}^{1}(1-r)^{\alpha q-1} M_{p}^{q}(f, r) d r\right)^{\frac{1}{q}}$. 
Accordingly, we shall denote by $H^{p, \alpha}(X)$ the space of functions $f \in \mathcal{H}(X)$ such that

$$
M_{p}(f, r)=O\left(\frac{1}{(1-r)^{\alpha}}\right) \quad(r \rightarrow 1),
$$

and set $\|f\|_{p, \alpha}=\sup _{0<r<1}(1-r)^{\alpha} M_{p}(f, r)$.

REMARK 1.3. The space $H^{p, p, \frac{1}{p}}(X)$ corresponds to the Bergman space $B^{p}(X)$ given by $X$-valued analytic functions such that $\int_{D}\|f(z)\|^{p} d A(z)<\infty$, where $d A(z)$ stands for the normalized Lebesgue measure on $D$.

Remark 1.4. Also observe that $B \operatorname{Bloch}(X)$, that is the space of $X$-valued analytic functions such that $\left\|f^{\prime}(z)\right\|=O\left(\frac{1}{1-|z|}\right)$, corresponds to functions with derivative in $H^{\infty, 1}(X)$.

Recall thaf $\operatorname{Bloch}(X)$ is a Banach space if we endow it with the norm

$$
\|f\|_{B l o c h(X)}=\max \left\{\|f(0)\|, \sup _{|z|<1}(1-|z|)\left\|f^{\prime}(z)\right\|\right\} .
$$

We start with some very elementary embeddings, well known to the experts on these spaces, whose proofs we include for completeness.

Lemma 1.1 (EmBeddings). Let $X$ be a complex banach space. If $1 \leq p, q \leq$ $\infty, 0<\alpha, p_{1} \leq p_{2}, q_{1} \leq q_{2}$ and $\alpha_{1} \leq \alpha_{2}$. Then

(i) $H^{p_{2}}(X) \subset H^{p_{2}, q_{1}, \alpha_{1}}(X) \subset H^{p_{1}, q_{2}, \alpha_{2}}(X) \subset H^{p_{1}, \alpha_{2}}(X)$.

(ii) $H^{p_{1}, q, \alpha}(X) \subset H^{p_{2}, q, \alpha+\frac{1}{p_{1}}-\frac{1}{p_{2}}}(X)$.

ProOF.

(i) The embedding $H^{p_{2}}(X) \subset H^{p_{2}, q_{1}, \alpha_{1}}(X)$ is obviuos.

For $H^{p_{1}, q_{2}, \alpha_{2}}(X) \subset H^{p_{1}, \alpha_{2}}(X)$ use

$$
\frac{1}{q \alpha} M_{p}^{q}(f, r)(1-r)^{q \alpha} \leq \int_{r}^{1}(1-s)^{\alpha q-1} M_{p}^{q}(f, s) d s
$$

To get $H^{p_{2}, q_{1}, \alpha_{1}}(X) \subset H^{p_{1}, q_{2}, \alpha_{2}}(X)$ use again (1.2):

Indeed, if $f \in H^{p_{2}, q_{1}, \alpha_{1}}(X)$ then $M_{p_{2}}(f, r) \leq \frac{C}{(1-r)^{\alpha_{1}}}$. Hence

$$
\begin{aligned}
\int_{0}^{1}(1-s)^{\alpha_{2} q_{2}-1} M_{p_{1}}^{q_{2}}(f, s) d s & \leq \int_{0}^{1}(1-s)^{\alpha_{1} q_{2}-1} M_{p_{2}}^{q_{2}}(f, s) d s \\
& \leq \int_{0}^{1}(1-s)^{\alpha_{1} q_{2}-1} M_{p_{2}}^{q_{1}}(f, s) \frac{C}{(1-s)^{\alpha_{1}\left(q_{2}-q_{1}\right)}} d s \\
& \leq\|f\|_{p_{2}, q_{1}, \alpha_{1}}^{q_{1}} .
\end{aligned}
$$

(ii) It follows from the following estimate (see $[\mathbf{D u}]$ )

$$
M_{p_{2}}\left(f, r^{2}\right) \leq C \frac{M_{p_{1}}(f, r)}{(1-r)^{\frac{1}{p_{1}}-\frac{1}{p_{2}}}},\left(p_{1} \leq p_{2}\right)
$$


Proposition 1.1. Let $1 \leq p \leq \infty$ and let $X$ be a complex Banach space.

(i) $f \in H^{p}(X) \Longrightarrow f^{\prime} \in H^{p}, 1(\bar{X})$.

(ii) If $0<\alpha \leq 1 \leq q$ then

$$
f^{\prime} \in H^{p, q, \alpha}(X) \Longrightarrow f \in H^{p}(X) .
$$

(iii) If $X \in(H)_{p}$ and $f \in H^{p}(X)$ then $f^{\prime} \in H^{p_{1}, q, \alpha}(X)$ for $p_{1} \leq p, \max \{p, 2\} \leq$ $q$ and $1 \leq \alpha$.

(iv) If $X \in(H)_{p}$ and $f \in H^{p}(X)$ then $f^{\prime} \in H^{p_{2}, \max \{p, 2\}, \alpha}(X)$ for $p \leq p_{2}$ and $1+\frac{1}{p}-\frac{1}{p_{2}} \leq \alpha$

Proof. (i) follows from the estimate

$$
M_{p}\left(f^{\prime}, r^{2}\right) \leq C \frac{M_{p}(f, r)}{1-r}
$$

(ii) It follows from the equality

$$
f\left(r e^{i t}\right)=\int_{0}^{r} f^{\prime}\left(s e^{i t}\right) d s+f(0)
$$

(iii) and (iv) are consequences from the definition and the Lemma 1.1.

Let us now compute the norm of $f(z)=\sum_{n=0}^{\infty} x_{n} z^{2^{n}}$ in $H^{p, q, \alpha}(X)$. The result is well known in the scalar-valued case but we include the proof here for sake of completeness.

Proposition 1.2. Let $1 \leq p \leq \infty, 1 \leq q<\infty$ and $0<\alpha \leq 1$ and let $f(z)=\sum_{n=0}^{\infty} x_{n} z^{2^{n}}$, where $x_{n} \in X$. Then

$$
\|f\|_{p, \alpha} \approx \sup _{n \in \mathbb{N}} 2^{-\alpha n}\left\|x_{n}\right\|
$$

and

$$
\|f\|_{p, q, \alpha} \approx\left(\sum_{n=0}^{\infty} 2^{-\alpha n q}|| x_{n} \|^{q}\right)^{\frac{1}{q}}
$$

Proof. Note that

$$
r^{2^{n}}\left\|x_{n}\right\| \leq M_{1}(f, r)
$$

and

$$
M_{\infty}(f, r) \leq \sum_{n=0}^{\infty}\left\|x_{n}\right\| r^{2^{n}}
$$

To get (1.5) assume first that $\sup _{n \in \mathbb{N}} 2^{-\alpha n}\left\|x_{n}\right\| \leq 1$; then we have, from (1.8),

$$
M_{p}(f, r) \leq M_{\infty}(f, r) \leq \sum_{n=0}^{\infty} 2^{\alpha n} r^{2^{n}} \leq C \sum_{n=0}^{\infty} n^{\alpha-1} r^{n} \leq \frac{C}{(1-r)^{\alpha}}
$$

On the other hand, if $M_{p}(f, r) \leq \frac{C}{(1-r)^{\alpha}}$ then (1.7) gives (taking $r=1-2^{-n}$ ) that

$$
\left(1-2^{-n}\right)^{2^{n}}\left\|x_{n}\right\| \leq C 2^{n \alpha},
$$


what shows that $\sup _{n \in \mathbb{N}} 2^{-\alpha n}\left\|x_{n}\right\| \leq C$.

To get (1.6) first use (1.7) and that

$$
\int_{1-2^{-n}}^{1-2^{-(n+1)}}(1-r)^{\alpha q-1} r^{2^{n} q} d r \approx 2^{-n \alpha q}
$$

to obtain

$$
\begin{aligned}
\left(\sum_{n=1}^{\infty} 2^{-\alpha n q}\left\|x_{n}\right\|^{q}\right)^{\frac{1}{q}} & \leq C\left(\sum_{n=0}^{\infty}\left(\int_{1-2^{-n}}^{1-2^{-(n+1)}}(1-r)^{\alpha q-1} r^{2^{n} q} d r\right)\left\|x_{n}\right\|^{q}\right)^{\frac{1}{q}} \\
& \leq C\left(\int_{0}^{1}(1-r)^{q \alpha-1} M_{p}^{q}(f, r) d r\right)^{\frac{1}{q}}=C\|f\|_{p, q, \alpha}
\end{aligned}
$$

To see the other inequality, consider the operator given by

$$
T\left(\left\{y_{n}\right\}\right)=(1-r)^{\alpha} \sum_{n=0}^{\infty} 2^{n \alpha} y_{n} r^{2^{n}} e^{2^{n} i t} .
$$

Note that (1.5) gives, for any $1 \leq p \leq \infty$, the boundedness of $T$ as an operator from $\ell^{\infty}(X)$ into $L^{\infty}\left(\frac{d r}{(1-r)}, L^{p}(\mathbb{T}, X)\right)$ (where, as usual, $L^{p}\left(\frac{d r}{1-r}, Y\right)$ stands for the space of $Y$-valued functions on $(0,1)$ that are $p$-integrable with respect to the measure $\left.\frac{d r}{1-r}\right)$.

It follows from (1.8) and a simple application of Fubbini's theorem that it is also bounded from $\ell^{1}(X)$ into $L^{1}\left(\frac{d r}{(1-r)}, L^{p}(\mathbb{T}, X)\right)$.

Now we use interpolation (see $[\mathbf{B L}])$ to get that

$$
T: l^{q}(X) \rightarrow L^{q}\left(\frac{d r}{(1-r)}, L^{p}(\mathbb{T}, X)\right)
$$

is bounded as well. What, in particular, implies that if $\sum_{n=0}^{\infty} 2^{-n \alpha q}\left\|x_{n}\right\|^{q}<\infty$ then $f \in H^{p, q, \alpha}(X)$.

REMARK 1.5. Let us mention that for the Bloch norm we have that

$$
\left\|\sum_{n=1}^{\infty} x_{n} z^{2^{n}}\right\|_{\operatorname{Bloch}(X)} \sim \sup _{n \in \mathbb{N}}\left\|x_{n}\right\| .
$$

This also follows from the scalar case (see $[\mathbf{A C P}, \mathbf{A S}]$ ) using the easy fact that

$$
\|f\|_{B \operatorname{Bloch}(X)}=\sup _{\left\|x^{*}\right\| \leq 1}\left\|x^{*} f\right\|_{B l o c h}
$$

Let us now mention some notions of geometry of Banach spaces that will be used in the sequel.

First recall that Kahane's inequalites can be stated as ([MPi, Pi1 ])

$$
\left\|\sum_{n \geq 0} x_{n} z^{2^{n}}\right\|_{p} \approx\left\|\sum_{n \geq 0} x_{n} z^{2^{n}}\right\|_{1}
$$

for any $0<p<\infty$. 
Given $2 \leq q<\infty$ a Banach space is said to have cotype $q$ (see [Pi2]) if there exists a constant $C>0$ such that, for any finite family $\left\{x_{n}\right\}_{n \geq 0}$ in $X$,

$$
\left(\sum_{n \geq 0}\left\|x_{n}\right\|^{q}\right)^{\frac{1}{q}} \leq C\left\|\sum_{n \geq 0} x_{n} z^{2^{n}}\right\|_{1} .
$$

Similarly for $1 \leq p \leq 2$ a Banach space is said to have type $p$ (see [Pi2]) if there exists a constant $C>0$ such that, for any finite family $\left\{x_{n}\right\}_{n \geq 0}$ in $X$,

$$
\left\|\sum_{n \geq 0} x_{n} z^{2^{n}}\right\|_{1} \leq C\left(\sum_{n \geq 0}\left\|x_{n}\right\|^{p}\right)^{\frac{1}{p}} .
$$

The notion of $K$-convex spaces (see $[\mathbf{P i 2}]$ ) can be also stated by looking at the projections onto lacunary sequences, that is the existence of a constant $C>0$ such that for some (or any) value of $1<p<\infty$

$$
\left\|\sum_{n \geq 0} x_{2^{n}} z^{2^{n}}\right\|_{p} \leq C\left\|\sum_{n \geq 0} x_{n} z^{n}\right\|_{p}
$$

for any sequence $x_{n} \in X$.

Another useful property for our purposes will be the notion of Fourier-type introduced by Peetre ([Pee $]$ ) which corresponds to spaces where the vector valued analogue of Hausdorff-Young's inequalies holds.

Let us recall that for $1 \leq p \leq 2$, a Banach space $X$ is said to have Fourier type $p$ if there exists a constant $C>0$ such that

$$
\left(\sum_{n=-\infty}^{\infty}\|\hat{f}(n)\|^{p^{\prime}}\right)^{\frac{1}{p^{\prime}}} \leq C\|f\|_{L^{p}(X)} .
$$

It is not hard to see that $X$ has Fourier type $p$ if and only if $X^{*}$ has Fourier type $p$. Typical examples are $L^{r}$ for $p \leq r \leq p^{\prime}$ or those obtained by interpolation between any Banach space and a Hilbert space.

Let us mention some results whose proofs can be seen in [AB2]

PROPOSITION 1.3.

(i) Let $q=\max \{p, 2\}$. If $X \in(H)_{p}$ then $X$ has cotype $q$.

(ii) If $H$ be a complex Hilbert space then $H \in(H)_{2}$.

(iii) Let $(\Omega, \Sigma, \mu)$ be a $\sigma$-finite measure space.

(a) If $p \geq 2$ and $p^{\prime} \leq q \leq p$ then $L^{q}(\mu) \in(H)_{p}$.

(b) If $1 \leq p \leq 2$ and $p \leq q \leq 2$ then $L^{q}(\mu) \in(H)_{p}$.

Let us finally recall the notion of vector valued $B M O A(X)$ (see $[\mathbf{B} 1]$ ) as the space of functions $f \in L^{1}(\mathbb{T}, X)$ with $\hat{f}(n)=0$ for $n<0$ such that

$$
\|f\|_{*, X}=\sup _{I} \frac{1}{|I|} \int_{I}\left\|f\left(e^{i t}\right)-f_{I}\right\| \frac{d t}{2 \pi}<\infty,
$$

where the supremum is taken over all intervals $I \in[0,2 \pi),|I|$ stands for the normalized Lebesgue measure of $I$ and $f_{I}=\frac{1}{|I|} \int_{I} f\left(e^{i t}\right) \frac{d t}{2 \pi}$.

The norm in the space is given by

$$
\|f\|_{B M O(X)}=\left\|\int_{-\pi}^{\pi} f\left(e^{i t}\right) \frac{d t}{2 \pi}\right\|+\|f\|_{*, X} .
$$


Let us point out certain results on the duality to be used later on. Although most of the results on the duality $H^{1}-B M O$ for vector valued functions (see $[\mathbf{B 2}$, Bo]) are given for the space $H^{1}$ defined in terms of atoms, it is easy to deduce from the known results the following facts:

For any Banach space $X$ one has that $B M O A\left(X^{*}\right)$ continuously embeds into $\left(H^{1}(X)\right)^{*}$. Actually if $f \in B M O A\left(X^{*}\right)$ and $g \in \mathcal{P}(X)$ then

$$
\left|\int_{0}^{1}<f\left(e^{i t}\right), g\left(e^{-i t}\right)>\frac{d t}{2 \pi}\right| \leq\|f\|_{B M O A\left(X^{*}\right)}\|g\|_{1, X} .
$$

If $X$ is a UMD space (see [B2]) then we actually have the validity of Fefferman's duality result

$$
\left(H^{1}(X)\right)^{*}=B M O A\left(X^{*}\right)
$$

\section{The theorem and its proof.}

Next result is known and part of the folklore we include here a proof because it will be a main point in our arguments.

Lemma 2.1. Let $1 \leq p, q \leq \infty, 0<\alpha$ and let $X$ be a complex Banach space.

Then $f$ belongs to $H^{p, q, \alpha}(X)$ if and only if $f^{\prime}$ belongs to $H^{p, q, \alpha+1}(X)$.

Proof. Let us assume $f \in H^{p, q, \alpha}(X)$. Denoting by $K(z)=1 /(1-z)^{2}$ the Bergman kernel, we can write $f^{\prime}=f * K$, therefore

$$
M_{p}\left(f^{\prime}, r^{2}\right) \leq M_{p}(f, r) M_{1}(K, r) \leq C \frac{M_{p}(f, r)}{(1-r)} .
$$

From this it easily follows that $f^{\prime} \in H^{p, q, \alpha+1}(X)$.

Conversely, let us assume $f^{\prime} \in H^{p, q, \alpha+1}(X)$ and that $f(0)=0$. To see that $f \in H^{p, q, \alpha}(X)$ it suffices to see that

$$
A=\left(\int_{\frac{1}{2}}^{1}(1-r)^{q \alpha-1} M_{p}^{q}(f, r) d r\right)^{\frac{1}{q}}<\infty .
$$

Observe first that

$$
f(z)=\int_{0}^{1} f^{\prime}(s z) d s
$$

This gives for $r \geq \frac{1}{2}$

$$
M_{p}(f, r) \leq \int_{0}^{1} M_{p}\left(f^{\prime}, r s\right) d s \leq 2 \int_{0}^{r} M_{p}\left(f^{\prime}, s\right) d s .
$$

To estimate $A$ we consider first the case $q=1$. Then

$$
\begin{aligned}
A & \leq 2 \int_{\frac{1}{2}}^{1}(1-r)^{\alpha-1} \int_{0}^{r} M_{p}\left(f^{\prime}, s\right) d s d r \\
& \leq 2 \int_{0}^{1} M_{p}\left(f^{\prime}, s\right)\left(\int_{s}^{1}(1-r)^{\alpha-1} d r\right) d s \\
& =(2 / \alpha)\left\|f^{\prime}\right\|_{p, 1, \alpha+1}^{q} .
\end{aligned}
$$



that

Assume now $q>1$. From Lemma 1.1 we have that $f^{\prime} \in H^{p, 1, \alpha+1}(X)$. Note

$$
A^{q} \leq \frac{2^{q}}{\alpha^{q}} \int_{\frac{1}{2}}^{1}(1-r)^{q \alpha-1}\left(\int_{0}^{r} M_{p}\left(f^{\prime}, s\right) d s\right)^{q} d r
$$

then integration by parts gives

$$
A^{q} \leq \frac{2^{q}}{\alpha^{q+1}} \int_{0}^{1}(1-r)^{q \alpha} M_{p}\left(f^{\prime}, r\right)\left(\int_{0}^{r} M_{p}\left(f^{\prime}, s\right) d s\right)^{q-1} d r .
$$

Now writting $(1-r)^{q \alpha}=(1-r)^{\alpha+1-1 / q}(1-r)^{(q-1) \alpha-1 / q^{\prime}}$, Hölder's inequality gives

$$
A^{q} \leq C\left(\int_{0}^{1}(1-r)^{q(\alpha+1)-1} M_{p}^{q}\left(f^{\prime}, r\right) d r\right)^{1 / q} A^{q / q^{\prime}},
$$

which is the desired inequality.

THEOREM 2.1. Let $1 \leq p_{1} \leq 2$ and $1 \leq p_{2}<\infty$ such that $\frac{1}{p_{1}}+\frac{1}{p_{2}} \geq 1$. Let $u: X \times Y \rightarrow Z$ be a bounded bilinear map where $X, Y, Z$ are complex Banach spaces. Let $1 \leq p, q \leq \infty$ are such that $\frac{1}{p}=\frac{1}{p_{1}}+\frac{1}{p_{2}}-1$ and $\frac{1}{q}=\frac{1}{2}+\frac{1}{\max \left\{p_{2}, 2\right\}}$. If $X \in(H)_{p_{1}}$ and $Y \in(H)_{p_{2}}$ then there exists a constant $C>0$ such that

$$
\left\|\left(f *_{u} g\right)^{\prime}\right\|_{p, q, 1} \leq C\|u\|\|f\|_{p_{1}}\|g\|_{p_{2}}
$$

for any $f(z)=\sum_{n=0}^{N} x_{n} z^{n} \in \mathcal{P}(X)$ and $g(z)=\sum_{n=0}^{M} y_{n} z^{n} \in \mathcal{P}(Y)$.

Proof. Let us first observe that if $S f(z)=z f(z)$ we have

$$
\begin{aligned}
{\left[S^{2}\left(f *_{u} g\right)\right]^{\prime \prime}(z) } & =\sum_{n=0}^{\infty} u\left((n+1) x_{n},(n+1) y_{n}\right) z^{n}+\sum_{n=0}^{\infty} u\left(x_{n},(n+1) y_{n}\right) z^{n} \\
& =\left[(S f)^{\prime}+f\right] *_{u}(S g)^{\prime}(z) .
\end{aligned}
$$

This, with the notation $f_{r}(z)=f(r z)$, means that

$$
\left[S^{2}\left(f *_{u} g\right)\right]^{\prime \prime}\left(r e^{i \theta}\right)=\left((S f)^{\prime}+f\right)_{r} *_{u}(S g)_{r}^{\prime}\left(e^{i \theta}\right) .
$$

Therefore, Young's theorem implies

$$
\left.M_{p}\left(\left[S^{2}\left(f *_{u} g\right)\right]^{\prime \prime}, r\right) \leq\|u\| M_{p_{1}}\left((S f)^{\prime}+f\right), r\right) M_{p_{2}}\left((S g)^{\prime}, r\right) .
$$

Now, denoting by $t=\max \left\{p_{2}, 2\right\}$ and $t^{\prime}$ such that $\frac{1}{t}+\frac{1}{t^{\prime}}=1$ then, using $\frac{1}{q}=\frac{1}{2}+\frac{1}{t}$, Hölder inequality gives that

$$
\begin{aligned}
& \left(\int_{0}^{1}(1-r)^{q\left(\frac{1}{2}+\frac{1}{t^{\prime}}\right)} M_{p}^{q}\left(S^{2}\left(f *_{u} g\right)^{\prime \prime}, r\right) d r\right)^{1 / q} \\
& \left.\leq\|u\|\left(\int_{0}^{1}(1-r) M_{p_{1}}^{2}\left((S f)^{\prime}+f\right), r\right) d r\right)^{1 / 2}\left(\int_{0}^{1}(1-r)^{t-1} M_{p_{2}}^{t}\left((S g)^{\prime}, r\right) r d r\right)^{1 / t} \\
& \leq C\|u\|\left(\|f\|_{p_{1}}+\left(\int_{0}^{1}(1-r) M_{p_{1}}^{2}\left((S f)^{\prime}, r\right) d r\right)^{1 / 2}\right)\left(\int_{0}^{1}(1-r)^{t-1} M_{p_{2}}^{t}\left((S g)^{\prime}, r\right) d r\right)^{1 / t} .
\end{aligned}
$$

Now using the properties $X \in(H)_{p_{1}}$ and $Y \in(H)_{p_{2}}$ we have that 


$$
\begin{aligned}
\left(\int_{0}^{1}(1-r)^{q\left(\frac{1}{2}+\frac{1}{t^{\prime}}\right)} M_{p}^{q}\left(S^{2}\left(f *_{u} g\right)^{\prime \prime}, r\right) d r\right)^{1 / q} & \leq C\|u\|\left(\|f\|_{p_{1}}+\|S f\|_{p_{1}}\right)\|S g\|_{p_{2}} \\
& \leq C\|u\|\|f\|_{p_{1}}\|g\|_{p_{2}} .
\end{aligned}
$$

Now observe that $q\left(\frac{1}{2}+\frac{1}{t^{\prime}}\right)=q\left(\frac{3}{2}-\frac{1}{t}\right)=2 q-1$ then from Lemma 2.1 we can estimate the norm of $S^{2}\left(f *_{u} g\right)^{\prime}$ in $H^{p, q, 1}(Z)$ by the norm of $S^{2}\left(f *_{u} g\right)^{\prime \prime}$ in $H^{p, q, 2}(Z)$ and hence

$$
\left\|\left(f *_{u} g\right)^{\prime}\right\|_{p, q, 1} \leq C\left\|u\left|\||| f\|_{p_{1}}\right| \mid g\right\|_{p_{2}} .
$$

TheOREm 2.2. Let $u: X \times Y \rightarrow Z$ be a bounded bilinear map where $X, Y, Z$ are complex Banach spaces. If $X \in(H)_{1}$ then there exists a constant $C>0$ such that

$$
\left\|\left(f *_{u} g\right)^{\prime}\right\|_{\infty, 2,1} \leq C \mid\|u\|\|f\|_{1}\|g\|_{B l o c h(Y)}
$$

for any $f(z)=\sum_{n=0}^{N} x_{n} z^{n} \in \mathcal{P}(X)$ and $g(z)=\sum_{n=0}^{M} y_{n} z^{n} \in \mathcal{P}(Y)$.

PRoOF. Same proof as before where $p_{1}=1, p_{2}=\infty$ gives the version of (2.1) in our case

$$
\left.M_{\infty}\left(\left[S^{2}\left(f *_{u} g\right)\right]^{\prime \prime}, r\right) \leq\|u\| M_{1}\left((S f)^{\prime}+f\right), r\right) M_{\infty}\left((S g)^{\prime}, r\right) .
$$

This implies that

$$
M_{\infty}\left(\left[S^{2}\left(f *_{u} g\right)\right]^{\prime \prime}, r\right) \leq C\|u\|\|g\|_{B \operatorname{loch}(Y)} \frac{\left.M_{1}\left((S f)^{\prime}+f\right), r\right)}{1-r} .
$$

Therefore

$$
\begin{aligned}
& \left(\int_{0}^{1}(1-r)^{3} M_{1}^{2}\left(S^{2}\left(f *_{u} g\right)^{\prime \prime}, r\right) d r\right)^{1 / 2} \\
& \left.\leq\|u\|\|g\|_{B l o c h(Y)}\left(\int_{0}^{1}(1-r) M_{1}^{2}\left((S f)^{\prime}+f\right), r\right) d r\right)^{1 / 2} \\
& \leq C\|u\|\|g\|_{B \operatorname{loch}(Y)}\left(\|f\|_{1}+\left(\int_{0}^{1}(1-r) M_{1}^{2}\left((S f)^{\prime}, r\right) d r\right)^{1 / 2}\right) \\
& \leq C\|u\|\|g\|_{B \operatorname{loch}(Y)}\left(\|f\|_{1}+\|S f\|_{1}\right) \\
& \leq C\|u\|\|g\|_{B \operatorname{loch}(Y)}\|f\|_{1} .
\end{aligned}
$$

In the applications of Theorems 2.1 and 2.2 that follow, sometimes polynomials are replaced by functions defined by power series. In all such cases the justification for doing so requires at most easy arguments, involving density of polynomials in the corresponding function space, that will be omitted.

Let us now give another way the see the improvement on $f *_{u} g$ from a different point of view.

Recall that for a function $f \in L^{p}(X)$ we can define the modulus of $p$-integrability

$$
\omega_{p}(f, t)=\sup _{|h| \leq t}\left(\int_{-\pi}^{\pi}\left\|f\left(e^{i(x+h)}\right)-f\left(e^{i x}\right)\right\|^{p} \frac{d x}{2 \pi}\right)^{\frac{1}{p}} .
$$


It is simple to see that $\omega_{p}(f, t)$ goes to zero as $t$ goes to zero. A bit more can be said for the functions with derivative in $H^{p, q, \alpha}(X)$ as the following lemma says.

Lemma 2.2. Let $1 \leq p \leq \infty, X$ a complex Banach space and $f: D \rightarrow X$ be analytic function with continuous extension to the boundary, still denoted by $f$.

Then $f^{\prime} \in H^{p, 1,1}(X)$ if and only if

$$
\int_{0}^{\pi} \omega_{p}(f, t) \frac{d t}{t}<\infty
$$

Proof. Asumme first that $f^{\prime} \in H^{p, 1,1}(X)$. Now if we fix $0<r<\rho<1$ and $x, h \in[-\pi, \pi)$ we have

$$
f\left(r e^{i(x+h)}\right)-f\left(r e^{i x}\right)=\int_{\Gamma} f^{\prime}(z) d z
$$

where the contour $\Gamma$ goes radially from $r e^{i x}$ to $\rho r e^{i x}$, then along $|z|=\rho r$ to $\rho r e^{i(x+h)}$ and again radially to $r e^{i(x+h)}$.

It follows that for any $\rho<1$

$\left\|f\left(r e^{i(x+h)}\right)-f\left(r e^{i x}\right)\right\| \leq \int_{\rho}^{1}\left\|f^{\prime}\left(r s e^{i x}\right)\right\| d s+\int_{\rho}^{1}\left\|f^{\prime}\left(r s e^{i(x+h)}\right)\right\| d s+\int_{x}^{x+h}\left\|f^{\prime}\left(\rho r e^{i y}\right)\right\| \frac{d y}{2 \pi}$.

It is easy to see (using the Hardy-Littlewood maximal theorem for $1<p<\infty$ ) that if $F(x)=\int_{x}^{x+h}\left\|f^{\prime}\left(\rho r e^{i y}\right)\right\| \frac{d y}{2 \pi}$ then $\|F\|_{p} \leq|h| M_{p}\left(f^{\prime}, \rho r\right)$.

Using now the previous estimate we can write

$$
\left(\int_{-\pi}^{\pi}\left\|f\left(r e^{i(x+h)}\right)-f\left(r e^{i x}\right)\right\|^{p} \frac{d x}{2 \pi}\right)^{\frac{1}{p}} \leq 2 \int_{\rho}^{1} M_{p}\left(f^{\prime}, r s\right) d s+|h| M_{p}\left(f^{\prime}, \rho r\right) .
$$

Since $M_{p}(f, r)$ is increasing we have for any $t>0$

$$
\omega_{p}(f, t) \leq 2 \int_{\rho}^{1} M_{p}\left(f^{\prime}, s\right) d s+t M_{p}\left(f^{\prime}, \rho\right) .
$$

Now taking $\rho=1-t$, dividing by $t$ and integrating we get

$$
\int_{0}^{\pi} \omega_{p}(f, t) \frac{d t}{t} \leq 2 \int_{0}^{1} \int_{1-t}^{1} M_{p}\left(f^{\prime}, s\right) d s d t+\int_{0}^{1} M_{p}\left(f^{\prime}, 1-t\right) d t \leq 3 \int_{0}^{1} M_{p}\left(f^{\prime}, r\right) d r .
$$

Conversely, using the Cauchy integral formula, we can write

$$
f^{\prime}\left(r e^{i x}\right)=\int_{-\pi}^{\pi} \frac{f\left(e^{i(x+h)}\right)-f\left(e^{i x}\right)}{\left(e^{i h}-r\right)^{2}} e^{i(h-x)} \frac{d h}{2 \pi} .
$$

From here it follows that

$$
M_{p}\left(f^{\prime}, r\right) \leq \int_{0}^{\pi} \frac{\omega_{p}(f, t)}{\left|e^{i t}-r\right|^{2}} d t
$$

Using the fact that $(1-r)^{2}+\left(C_{0} t\right)^{2} \leq\left|e^{i t}-r\right|^{2}$ for certain constant $C_{0}>0$ we have that

$$
\int_{0}^{1} M_{p}\left(f^{\prime}, r\right) d r \leq \int_{0}^{1} \int_{0}^{\pi} \frac{\omega_{p}(f, t)}{(1-r)^{2}+\left(C_{0} t\right)^{2}} d t d r .
$$

Finally applying Fubini and the change of variable $1-r=\left(C_{0} t\right) s$ we get 


$$
\int_{0}^{1} M_{p}\left(f^{\prime}, r\right) d r \leq C \int_{0}^{\pi} \omega_{p}(f, t) \frac{d t}{t} . \square
$$

Corollary 2.1. Let $1 \leq p \leq 2$ and let $u: X \times Y \rightarrow Z$ be a bounded bilinear map where $X, Y, Z$ are complex Banach spaces. Assume $X \in(H)_{1}$ and $Y \in(H)_{p}$. If $f \in H^{1}(X)$ and $g \in H^{p}(Y)$ then

$$
\int_{0}^{\pi} \omega_{p}\left(f *_{u} g, t\right) \frac{d t}{t}<\infty .
$$

Proof. It follows from the application of Theorem 2.1 and Lemma 2.2 consecutively.

Our next aim is to get information on Taylor coefficients of $f *_{u} g$. For that purpose the following lemma is useful.

Lemma 2.3. Let $1 \leq q \leq \infty, 0<\alpha$ and $X$ a complex Banach space.

If $f(z)=\sum_{n=0}^{\infty} x_{n} z^{n} \in H^{1, q, \alpha}(X)$ then

$$
\left(\sum_{n=0}^{\infty} 2^{-n \alpha q} \sup _{2^{n} \leq k<2^{n+1}}\left\|x_{n}\right\|^{q}\right)^{\frac{1}{q}} \leq C\|f\|_{1, q, \alpha}
$$

Proof. Note that

$$
\sup _{2^{n} \leq k<2^{n+1}}\left\|x_{k}\right\| \leq C M_{1}\left(f, 1-2^{-n}\right) .
$$

Therefore

$$
\begin{aligned}
\|f\|_{1, q, \alpha} & =\left(\sum_{n=0}^{\infty} \int_{1-2^{-n}}^{1-2^{-(n+1)}}(1-r)^{q \alpha-1} M_{1}^{q}(f, r) d r\right)^{1 / q} \\
& \left.\geq C \sum_{n=0}^{\infty} M_{1}^{q}\left(f, 1-2^{-n}\right) \int_{1-2^{-n}}^{1-2^{-(n+1)}}(1-r)^{q \alpha-1} d r\right)^{1 / q} \\
& \geq C\left(\sum_{n=0}^{\infty} 2^{-n \alpha q} \sup _{2^{n} \leq k<2^{n+1}}\left\|x_{n}\right\|^{q}\right)^{\frac{1}{q}} .
\end{aligned}
$$

Corollary 2.2. Let $u: X \times Y \rightarrow Z$ be a bounded bilinear map where $X, Y, Z$ are complex Banach spaces. Let $f \in H^{1}(X)$ and $g \in H^{1}(Y)$ where $X, Y \in(H)_{1}$. Then

$$
\sum_{n=0}^{\infty} \sup _{2^{n} \leq k<2^{n+1}}\left\|u\left(x_{k}, y_{k}\right)\right\| \leq C\|u\|\|f\|_{1}\|g\|_{1}
$$

In particular

$$
\sum_{n=0}^{\infty} \frac{\left\|u\left(x_{n}, y_{n}\right)\right\|}{n+1} \leq C\|u\|\||| f\|_{1}\|g\|_{1}
$$


Proof. Note that in this case Theorem 2.1 gives that $h^{\prime}(z)=\left(f *_{u} g\right)^{\prime}(z)=$ $\sum_{n=1}^{\infty} n u\left(x_{n}, y_{n}\right) z^{n-1} \in H^{1,1,1}$. Now apply Lemma 2.3 .

Next result is known but we include the proof for completeness.

Lemma 2.4. Let $0<\gamma \leq 1, \beta>0$ and $\alpha_{n} \geq 0$. Then if $I_{n}=\mathbb{N} \cap\left[2^{n}, 2^{n+1}\right)$ we have

$$
\int_{0}^{1}(1-r)^{\beta-1}\left(\sum_{n=0}^{\infty} \alpha_{n} r^{n}\right)^{\gamma} d r \approx \sum_{n=0}^{\infty} 2^{-n \beta}\left(\sum_{k \in I_{n}} \alpha_{k}\right)^{\gamma} .
$$

PROOF.

$$
\begin{aligned}
\int_{0}^{1}(1-r)^{\beta-1}\left(\sum_{n=0}^{\infty} \alpha_{n} r^{n}\right)^{\gamma} d r & =\sum_{n=0}^{\infty} \int_{1-2^{-n}}^{1-2^{-(n+1)}}(1-r)^{\beta-1}\left(\sum_{n=0}^{\infty} \alpha_{n} r^{n}\right)^{\gamma} d r \\
& \geq C \sum_{n=0}^{\infty} 2^{-n(\beta-1)} \int_{1-2^{-n}}^{1-2^{-(n+1)}}\left(\sum_{k \in I_{n}} \alpha_{k} r^{k}\right)^{\gamma} d r \\
& \geq C \sum_{n=0}^{\infty} 2^{-n(\beta-1)}\left(\sum_{k \in I_{n}} \alpha_{k}\right)^{\gamma} \int_{1-2^{-n}}^{1-2^{-(n+1)}} r^{2^{n} \gamma} d r \\
& \geq C \sum_{n=0}^{\infty} 2^{-n \beta}\left(\sum_{k \in I_{n}} \alpha_{k}\right)^{\gamma}
\end{aligned}
$$

Let us now show the converse inequality. Using that $\gamma \leq 1$ we have

$$
\begin{aligned}
\int_{0}^{1}(1-r)^{\beta-1}\left(\sum_{n=0}^{\infty} \alpha_{n} r^{n}\right)^{\gamma} d r & \leq C \int_{0}^{1}(1-r)^{\beta-1}\left(\sum_{n=0}^{\infty}\left(\sum_{k \in I_{n}} \alpha_{k}\right) r^{2^{n}-1}\right)^{\gamma} d r \\
& \leq C \int_{0}^{1}(1-r)^{\beta-1}\left(\sum_{n=0}^{\infty}\left(\sum_{k \in I_{n}} \alpha_{k}\right)^{\gamma} r^{\left(2^{n}-1\right) \gamma}\right) d r \\
& \leq C \sum_{n=0}^{\infty} \int_{0}^{1}(1-r)^{\beta-1} r^{2^{n} \gamma-1}\left(\sum_{k \in I_{n}} \alpha_{k}\right)^{\gamma} d r \\
& \leq C \sum_{n=0}^{\infty} B\left(\beta, 2^{n} \gamma\right)\left(\sum_{k \in I_{n}} \alpha_{k}\right)^{\gamma} \\
& \leq C \sum_{n=0}^{\infty} 2^{-n \beta}\left(\sum_{k \in I_{n}} \alpha_{k}\right)^{\gamma}
\end{aligned}
$$

Corollary 2.3. Let $1 \leq p_{1} \leq 2,1 \leq p_{2} \leq \infty$ such that $\frac{3}{2} \leq \frac{1}{p_{1}}+\frac{1}{p_{2}}<2$. Let $u: X \times Y \rightarrow Z$ be a bounded bilinear map where $X, Y, Z$ are complex Banach spaces. Let $f \in H^{p_{1}}(X)$ and $g \in H^{p_{2}}(Y)$. Assume $X \in(H)_{p_{1}}$ and $Y \in(H)_{p_{2}}$ and $Z$ has Fourier type $p$ where $\frac{1}{p}=\frac{1}{p_{1}}+\frac{1}{p_{2}}-1$ then

$$
\left(\sum_{n=0}^{\infty}\left(\sum_{k=2^{n}}^{2^{n+1}}\left\|u\left(x_{k}, y_{k}\right)\right\|^{p^{\prime}}\right)^{\frac{q}{p^{\prime}}}\right)^{\frac{1}{q}} \leq C\|u\|\|f\|_{p_{1}}\|g\|_{p_{2}}
$$


for $\frac{1}{q}=\frac{1}{2}+\frac{1}{\max \left\{2, p_{2}\right\}}$.

Proof. It follows from Theorem 2.1 that $h^{\prime}(z)=\left(f *_{u} g\right)^{\prime}(z)=\sum_{n=1}^{\infty} n u\left(x_{n}, y_{n}\right) z^{n-1} \in$ $H^{p, q, 1}(Z)$. Using that $Z$ has Fourier type $p$ (note that the restriction gives $1<p \leq$ 2) we have, from (1.11), that

$$
\left(\sum_{n=0}^{\infty} n^{p^{\prime}}\left\|u\left(x_{n}, y_{n}\right)\right\|^{p^{\prime}} r^{p^{\prime}(n-1)}\right)^{\frac{1}{p^{\prime}}} \leq C M_{p}\left(\left(f *_{u} g\right)^{\prime}, r\right) .
$$

Therefore an application of Lemma 2.4 with $\alpha_{n}=n^{p^{\prime}}\left\|u\left(x_{n}, y_{n}\right)\right\|^{p^{\prime}}, \beta=q$ and $\gamma=\frac{q}{p^{\prime}} \leq 1$ gives the result.

Corollary 2.4. Let $1<p_{1} \leq p_{2} \leq \infty$ such that $\frac{1}{p_{1}}+\frac{1}{p_{2}} \geq 1$. Let $u$ : $X \times Y \rightarrow Z$ be a bounded bilinear map where $X, Y, Z$ are complex Banach spaces. Let $f \in H^{p_{1}}(X)$ and $g \in H^{p_{2}}(Y)$ where $X \in(H)_{p_{1}}$ and $Y \in(H)_{p_{2}}$ and either $X$ or $Y$ is $K$-convex then

$$
\left(\sum_{n=0}^{\infty}\left\|u\left(x_{2^{n}}, y_{2^{n}}\right)\right\|^{q}\right)^{\frac{1}{q}} \leq C\|u\|\|f\|_{p_{1}}\|g\|_{p_{2}}
$$

where $\frac{1}{q}=\frac{1}{2}+\frac{1}{\max \left\{p_{2}, 2\right\}}$.

Proof. Assume that $X$ is $K$-convex (similar proof works for $Y$ ). Take $f(z)=$ $\sum_{n=0}^{\infty} x_{n} z^{n}, g(z)=\sum_{n=0}^{\infty} y_{n} z^{n}$ and apply Theorem 2.1 with $f_{1}(z)=\sum_{n=0}^{\infty} x_{2^{n}} z^{2^{n}}$ and $g$. Note that $\left(f_{1} *_{u} g\right)^{\prime}(z)=\sum_{n=0}^{\infty} 2^{n} u\left(x_{2^{n}}, y_{2^{n}}\right) z^{2^{n}-1} \in H^{p, q, 1}(Z)$. Now apply Proposition 1.2 and the estimate given by (1.10) $\left\|f_{1}\right\|_{p_{1}} \leq C\|f\|_{p_{1}}$ to finish the proof.

REMARK 2.1. The case $p_{1}=1$ in the previous Corollary 2.4 can be obtained if we assume that the Banach space $X$ satisfies that the Paley projection is bounded in $H^{1}(X)$. This property has been considered in $[\mathbf{L - P P}]$. And, for instance, $X=L^{1}(\mu)$ or the nuclear operators $\sigma_{1}$ (see Section 3 ) satisfy such a property.

\section{Applications to Schatten classes.}

Given $1 \leq p<\infty$ we shall denote by $\sigma_{p}$ the Banach space of compact operators on $l^{2}$ such that

$$
\|A\|_{\sigma_{p}}=\left(\operatorname{tr}\left(A^{*} A\right)^{\frac{p}{2}}\right)^{\frac{1}{p}}<\infty .
$$

It is well known that $\sigma_{1}$ coincides with the space of nuclear operators on $l^{2}$ and $\sigma_{2}$ with the space of Hilbert-Schmidt operators on $l^{2}$. The reader is referred to $[\mathbf{G K}]$ for general properties on $\sigma_{p}$.

LEMMA 3.1. (see [B1])

If $1 \leq p \leq 2$ then $\sigma_{p}$ has the $(H)_{1}$-property.

To cover other values of $p$ we shall use some of the recent advances on interpolation of vector-valued Hardy spaces. It is known (see $[\mathbf{B X}]$ ) that interpolation spaces by complex or real method, $\left(H^{p_{1}}\left(X_{1}\right), H^{p_{2}}\left(X_{2}\right)\right)_{\theta}$ or $\left(H^{p_{1}}\left(X_{1}\right), H^{p_{2}}\left(X_{2}\right)\right)_{\theta, p}$ do not coincide, in general, with $H^{p_{\theta}}\left(X_{\theta}\right)$ or $H^{p_{\theta}}\left(X_{\theta, p}\right)$, but nevertheless there are some positive results for some particular spaces, like $L^{p}$ or $\sigma_{p}$, where the expected result remains true (see $[\mathbf{X} \mathbf{1}, \mathbf{X} \mathbf{2}, \mathbf{B X}, \mathbf{P i 3}]$ ). 
Proposition 3.1. Let $1 \leq p<\infty$.

(i) If $p \geq 2$ and $p^{\prime} \leq q \leq p$ then $\sigma_{q} \in(H)_{p}$.

(ii) If $1 \leq p \leq 2$ and $p \leq q \leq 2$ then $\sigma_{q} \in(H)_{p}$.

Proof. Observe that the $(H)_{p}$ property can be stated in terms of the boundedness of the operator $T: H^{p}(X) \rightarrow L^{\max \{2, p\}}\left(\frac{d r}{1-r}, L^{p}(\mathbb{T}, X)\right)$ given by

$$
T(f)(r, t)=(1-r) f^{\prime}\left(r e^{i t}\right) .
$$

Note first that

$$
T: H^{2}\left(\sigma_{2}\right) \rightarrow L^{2}\left(\frac{d r}{1-r}, L^{2}\left(\mathbb{T}, \sigma_{2}\right)\right)
$$

is bounded by Proposition 1.3 part (ii).

To see (i), choose $\theta=1-\frac{2}{p}$ and $s=\theta\left(\frac{1}{q}-\frac{1}{p}\right)^{-1}$, so that $\frac{1}{p}=\frac{1-\theta}{2}$ and $\frac{1}{q}=\frac{1-\theta}{2}+\frac{\theta}{s}$, which gives

$$
\left[H^{2}\left(\sigma_{2}\right), \operatorname{BMOA}\left(\sigma_{s}\right)\right]_{\theta}=H^{p}\left(\sigma_{q}\right)
$$

and

$$
\left[L^{2}\left(\frac{d r}{1-r}, L^{2}\left(\mathbb{T}, \sigma_{2}\right)\right), L^{\infty}\left(\frac{d r}{1-r}, L^{\infty}\left(\mathbb{T}, \sigma_{s}\right)\right)\right]_{\theta}=L^{p}\left(\frac{d r}{1-r}, L^{p}\left(\mathbb{T}, \sigma_{q}\right)\right) .
$$

In order to interpolate, just note that $B M O A(X) \subset B \operatorname{loch}(X)$ for any $X$, so

$$
T: B M O A\left(\sigma_{s}\right) \rightarrow L^{\infty}\left(\frac{d r}{1-r}, L^{\infty}\left(\mathbb{T}, \sigma_{s}\right)\right)
$$

is bounded for any value $1 \leq s \leq \infty$.

To see (ii), let $\theta$ be such that $\frac{1}{p}=1-\frac{\theta}{2}$ and $s$ such that $\frac{1}{q}=\frac{1-\theta}{s}+\frac{\theta}{2}$. Then

$$
\left.\left[H^{1}\left(\sigma_{s}\right), H^{2}\left(\sigma_{2}\right)\right)\right]_{\theta}=H^{p}\left(\sigma_{q}\right)
$$

and

$$
\left[L^{2}\left(\frac{d r}{1-r}, L^{1}\left(\mathbb{T}, \sigma_{s}\right)\right), L^{2}\left(\frac{d r}{1-r}, L^{2}\left(\mathbb{T}, \sigma_{2}\right)\right)\right]_{\theta}=L^{2}\left(\frac{d r}{1-r}, L^{p}\left(\mathbb{T}, \sigma_{q}\right)\right) .
$$

It follows from our assumptions that $1 \leq s \leq 2$ and then, using Lemma 3.1, $\sigma_{s} \in(H)_{1}$. Hence we get $\sigma_{q} \in(H)_{p}$.

TheOREm 3.1. Let $1 \leq p \leq 2$. There exists a constant $C>0$ such that if $\left\{T_{n}\right\}$ is a sequence of operators in $\sigma_{p}$ and if $\left\{y_{n}\right\}$ is a sequence of vectors in $l^{2}$ such that $\sum_{n=0}^{\infty}\left\|y_{n}\right\|^{2}=1$ then

$$
\sum_{n=0}^{\infty}\left(\sum_{k=2^{n}}^{2^{n+1}}\left\|T_{k}\left(y_{k}\right)\right\|^{2}\right)^{\frac{1}{2}} \leq C \frac{1}{2 \pi} \int_{-\pi}^{\pi}\left\|\sum_{n=0}^{\infty} T_{n} e^{i n t}\right\|_{\sigma_{p}} d t .
$$

Proof. Consider $f(z)=\sum_{n=0}^{\infty} T_{n} z^{n} \in H^{1}\left(\sigma_{p}\right)$ and $g(z) \sum_{n=0}^{\infty} y_{n} z^{n} \in H^{2}\left(l^{2}\right)$. Now apply Corollary 2.3 for the cases $p_{1}=1, p_{2}=2, X=\sigma_{p}$ and $Y=l^{2}$ with the bilinear map $u: \sigma_{p} \times l^{2} \rightarrow l^{2}$ given by $u(T, y)=T(y)$. 


\section{Applications to convolution of sequences.}

In this section we consider the bilinear map given by Young's theorem, that is for $\frac{1}{p}+\frac{1}{q} \geq 1$ and $\frac{1}{r}=\frac{1}{p}+\frac{1}{q}-1$ we have the bounded bilinear map $u$ : $L^{p}(\mathbb{R}) \times L^{q}(\mathbb{R}) \rightarrow L^{r}(\mathbb{R})$ given by $u(f, g)=f * g$. The reader is referred to [ AB1, AB2 ] for particular cases and some applications.

TheOREm 4.1. Let $1 \leq p_{1} \leq 2,1 \leq p_{2}<\infty$ such that $\frac{1}{p_{1}}+\frac{1}{p_{2}} \geq 1$. If $\frac{1}{p_{1}}+\frac{1}{p_{2}}-1=\frac{1}{p}$ and $\frac{1}{2}+\frac{1}{\max \left\{p_{2}, 2\right\}}=\frac{1}{q}$ then, we have

$$
\left(\sum_{n=0}^{\infty}\left\|f_{n} * g_{n}\right\|_{p}^{q}\right)^{\frac{1}{q}} \leq C\left\|\left(\sum_{n=1}^{\infty}\left|f_{n}\right|^{2}\right)^{1 / 2}\right\| p_{p_{1}}\left\|\left(\sum_{n=1}^{\infty}\left|g_{n}\right|^{2}\right)^{1 / 2}\right\|_{p_{2}}
$$

for $f_{n} \in L^{p_{1}}(\mathbb{R}), g_{n} \in L^{p_{2}}(\mathbb{R})$.

Proof. Consider $f(z)=\sum_{n=0}^{\infty} f_{n} z^{2^{n}}$ and $g(z)=\sum_{n=0}^{\infty} g_{n} z^{2^{n}}$.

It is a well-known consequence of Kintchine's inequalities that

$$
\|f\|_{H^{p_{1}\left(L^{p_{1}}\right)}} \sim\left\|\left(\sum_{n=1}^{\infty}\left|f_{n}\right|^{2}\right)^{1 / 2}\right\|_{p_{1}} \text { and }\|g\|_{H^{p_{2}\left(L^{p_{2}}\right)}} \sim\left\|\left(\sum_{n=1}^{\infty}\left|g_{n}\right|^{2}\right)^{1 / 2}\right\|_{p_{2}} .
$$

The result now follows from Theorem 2.1 and Proposition 1.2 applied to $\left(f *_{u}\right.$ $g)^{\prime}(z)=\sum_{n=0}^{\infty} 2^{n} f_{n} * g_{n} z^{2^{n}-1} \in H^{p, q, 1}\left(L^{p}(\mathbb{R})\right)$.

REMARK 4.1. The reader should observe that this previous result can also be achieved by using Hölder's inequality and the cotype of the spaces appearing in the theorem.

TheOrem 4.2. Let $1 \leq p_{1} \leq 2,1 \leq p_{2} \leq \infty$ such that $1 \leq \frac{1}{p_{1}}+\frac{1}{p_{2}} \leq \frac{3}{2}$. If $\frac{1}{p_{1}}+\frac{1}{p_{2}}-1=\frac{1}{p}$. Then we have

$$
\left\|\left(\sum_{n=1}^{\infty}\left|f_{n} * g_{n}\right|^{2}\right)^{1 / 2}\right\|\left\|_{p} \leq C\right\|\left(\sum_{n=1}^{\infty}\left|f_{n}\right|^{2}\right)^{1 / 2}\|\|_{p_{1}} \sup _{n \in \mathbb{N}}\left\|g_{n}\right\|_{p_{2}}
$$

for $f_{n} \in L^{p_{1}}(\mathbb{R}), g_{n} \in L^{p_{2}}(\mathbb{R})$.

Proof. As above consider $f(z)=\sum_{n=0}^{\infty} f_{n} z^{2^{n}}$ and $g(z)=\sum_{n=0}^{\infty} g_{n} z^{2^{n}}$.

Using Remark 1.5 we have that

$$
\|g\|_{\text {Bloch }\left(L^{p_{2}}\right)} \sim \sup _{n \in \mathbb{N}}\left\|g_{n}\right\|_{p_{2}} .
$$

Therefore Theorem 2.2 and Proposition 1.2 give that

$$
\left(\sum_{n=0}^{\infty}\left\|f_{n} * g_{n}\right\|_{p}^{2}\right)^{\frac{1}{2}} \leq C\left\|\left(\sum_{n=1}^{\infty}\left|f_{n}\right|^{2}\right)^{1 / 2}\right\|_{p_{1}} \sup _{n \in \mathbb{N}}\left\|g_{n}\right\|_{p_{2}}
$$

Now using that $p \geq 2$ we apply that $L^{p}$ has type 2 to get

$$
\left\|\left(\sum_{n=1}^{\infty}\left|f_{n} * g_{n}\right|^{2}\right)^{1 / 2}\right\|_{p} \leq\left(\sum_{n=0}^{\infty}\left\|f_{n} * g_{n}\right\|_{p}^{2}\right)^{\frac{1}{2}}
$$

and the proof is finished. 


\section{Applications to vector valued multipliers.}

In this section we deal with the following natural problem in the vector-valued setting. Given two Banach spaces $X, Y$, a sequence $\left\{x_{n}\right\} \in X$ and a sequence of opertators $\left\{T_{n}\right\} \in L(X, Y)$ we want to study the map from $\mathcal{H}(X)$ to $\mathcal{H}(Y)$ defined by $f(z)=\sum_{n=0}^{\infty} x_{n} z^{n} \rightarrow g(z)=\sum_{n=0}^{\infty} T_{n}\left(x_{n}\right) z^{n}$. One wants to find the conditions on $\left\{T_{n}\right\} \in L(X, Y)$ to get this map bounded from one space of $X$-valued functions into another space of $Y$-valued functions. The reader is referred to [B1] where the case $H^{1}(X)$ into $B M O A(Y)$ is analyzed.

Here we present an alternative proof of some result in $[\mathbf{B} 1]$.

TheOrem 5.1. Let $1 \leq p \leq 2 \leq q<\infty$ and let $T_{n}: l^{p} \rightarrow l^{q}$ be a sequence of bounded linear operators. If $\sum_{n=0}^{\infty} T_{n} z^{n} \in \operatorname{Bloch}\left(L\left(l^{p}, l^{q}\right)\right)$ and $\sum_{n=0}^{\infty} x_{n} z^{n} \in$ $H^{1}\left(l^{p}\right)$ then $\sum_{n=0}^{\infty} T_{n}\left(x_{n}\right) z^{n} \in B M O A\left(l^{q}\right)$.

Proof. Taking into account that $\left(H^{1}\left(l^{q^{\prime}}\right)\right)^{*}=B M O A\left(l^{q}\right)($ since $2 \leq q<\infty)$ it suffices to see that

$$
\left|\sum_{n=0}^{\infty}<T_{n}\left(x_{n}\right), y_{n}>\right| \leq C\left\|\sum_{n=0}^{\infty} x_{n} z^{n}\right\|_{1}\left\|\sum_{n=0}^{\infty} y_{n} z^{n}\right\|_{1}
$$

for any $\sum_{n=0}^{\infty} y_{n} z^{n} \in H^{1}\left(l^{q^{\prime}}\right)$.

Let us consider the natural embedding $u: l^{p} \times l^{q^{\prime}} \rightarrow l^{p} \hat{\otimes} l^{q^{\prime}}$ and recall that $L\left(l^{p}, l^{q}\right)$ is naturally identified to $\left(l^{p} \hat{\otimes} l^{q^{\prime}}\right)^{*}$ under the pairing $[T, x \otimes y]=<T(x), y>$.

Therefore

$$
\left|\sum_{n=0}^{\infty}<T_{n}\left(x_{n}\right), y_{n}>\right|=\left|\sum_{n=0}^{\infty}\left[T_{n}, x_{n} \otimes y_{n}\right]\right|=\left|\sum_{n=0}^{\infty}\left[T_{n}, u\left(x_{n}, y_{n}\right)\right]\right|
$$

Now we recall that $\operatorname{Bloch}\left(X^{*}\right)$ corresponds, under the pairing $\sum_{n=0}^{\infty}<x_{n}, x_{n}^{*}>$, to the dual of the space of functions whose derivative is in $H^{1,1,1}(X)$.

The combination of this facts and Theorem 2.2 applied to $p_{1}=p_{2}=1$ and $X=l^{p}$ and $Y=l^{q^{\prime}}$ which belong to $(H)_{1}$ gives the desired result.

\section{References}

[A] H. Amann, Operator-valued Fourier multipliers, vector valued Besov spaces and applications, Math. Nachr. 186 (1997), 15-56.

[ACP] J.M. Anderson, J. Clunie. Ch Pommerenke, On Bloch functions and normal functions, J. Reine Angew. Math. 270 (1974), 12-37.

[AS] J.M. Anderson, A.L. Shields, Coefficient multipliers on Bloch functions, Trans. Amer. Math. Soc. 224 (1976), 256-265.

[AB1] J.L. Arregui, O. Blasco, On the Bloch space and convolution of functions in the $L^{p}$-valued case., Collectanea Math. 48 (1997), 363-373.

[AB2] J.L. Arregui, O. Blasco, Convolution of three functions by means of bilinear maps and applications, Illinois J 43 (1999).

[BL] J. Berg and J. Lofstrom, Interpolation spaces. An introduction, Springer-Verlag, Berlin and New York, 1973.

[B1] O. Blasco, Vector valued analytic functions of bounded mean oscillation and geometry of Banach spaces, Illinois J. 41 (1997), 532-557.

[B2] O. Blasco, Hardy spaces of vector valued functions: Duality, Trans. Amer. Math. Soc. 308 (1988), 495-507.

[BP] O. Blasco and A. Pelczynski, Theorems of Hardy and Paley for vector valued analytic functions and related classes of Banach spaces, Trans. Amer. Math. Soc. 323 (1991), $335-367$. 
[BX] O. Blasco and Q. Xu, Interpolation between vector valued Hardy spaces, J. Funct. Anal. 102 (1991), 331-359.

[Bo] J. Bourgain, Vector valued singular integrals and the $H^{1}-B M O$ duality, Probability theory and Harmonic Analysis (1986), Marcel Decker, New York, 1-19.

[D] P. Duren, Theory of $H_{p}$-spaces, Academic Press, New York, 1970.

[F1] T.M. Flett, On the rate of growth of mean values of holomorphic and harmonic functions, Proc. London Math. Soc. 20 (1970), 749-768.

[F2] T.M. Flett, Lipschizt spaces of functions on the circle and the disc, J. Math. Anal. Appl. 39 (1972), 125-158.

[F3] T.M. Flett, The dual of an inequality of Hardy and Littlewood and some related inequalities, ibid. 38 (1972), 746-765.

[GR] J. Garcia-Cuerva and J.L. Rubio de Francia, Weigthed norm inequalities and related topics, North-Holland, Amsterdam, 1985.

[G] J. B. Garnett, Bounded analytic functions, Academic Press, New York, 1981.

[GK] I.C. Gohberg and M.G. Krein, Theory and applications of Volterra operators in Hilbert space, Trans. Math. Monographs Amer. Math. Soc., vol. 24, Providence, R.I., 1970.

[HL1] G.H. Hardy, J.E. Littlewood, Some properties of fractional integrals II, Math. Z. 34 (1932), 403-439.

[HL2] G.H. Hardy, J.E. Littlewood, Theorems concerning mean values of analytic or harmonic functions, Quart. J. Math. 12 (1941), 221-256.

[HL3] G.H. Hardy, J.E. Littlewood, Notes on the theory of series (XX) Generalizations of a theorem of Paley, Quart. J. Math. 8 (1937), 161-171.

[LT] J. Lindenstrauss, L. Tzafriri, Classical Banach spaces II, Springer Verlag, New York, 1979.

[LP] J.E. Littlewood and R.E.A.C. Paley, Theorems on Fourier series and power series (II), Proc. London Math. Soc. 42 (1936), 52-89.

[L-PP] F. Lust-Piquard and G. Pisier, Non commutative Kinthine and Paley inequalities, Ark. Math. 29 (1991), 241-260.

[MP] M. Mateljevic, M. Pavlovic, Multipliers of $H^{p}$ and BMO, Pacific J. Math. 146 (1990), 71-84.

[MPi] B. Maurey, G Pisier, Séries de variables aléatories vectorialles independentes et proprietés geometriques des espaces de Banach, Studia Math. 58 (1976), 45-90.

[Pee] J.Peetre, Sur la transformation de Fourier des fonctions a valeurs vectorielles, Rend. Sem. Mat. Univ. Padova 42 (1969), 15-46.

[Pe] A. Pelczynski, On commesurate sequences of characters, Proc. Amer. Math. Soc. 104 (1988), 525-531.

[Pi1] G. Pisier, Les inegalités de Kintchine-Kahane d'apres C. Borel, Exposé VII, Ecole Polytechnique, Centre de Matematiques., Séminaire sur le Géometrie d'Espaces de Banach (1977-1978).

[Pi2] G. Pisier, Factorization of linear operators and geometry of Banach spaces, vol. 60, AMS Regional Conference Series in Math., 1986.

[Pi3] G. Pisier, Interpolation between $H^{p}$ spaces and non-commutative generalizations.I, Pacific J. of Math. 155 (1992), 341-368.

[X1] Q. Xu, Applications du theorem de factorisation pour des fonctions a valeurs operateurs, Studia Math. 95 (1990), 273-291.

[X2] Q. Xu, Real interpolation of some Banach lattices valued Hardy spaces, Bull. Sc. Math. 2 série 116 (1992), 227-246.

[Z] A. Zygmund, Trigonometric series, Cambrigde Univ. Press., New York, 1959.

Oscar Blasco. Departamento de Análisis Matemático, Universidad de Valencia, 46100 BurJassot (VAlencia), Spain.

E-mail address: oblasco@uv.es 